\title{
Development of a surface plasmon resonance and nanomechanical biosensing hybrid platform for multiparametric reading
}

\author{
Mar Alvarez, ${ }^{1, a)}$ David Fariña, ${ }^{1}$ Alfonso M. Escuela,${ }^{2}$ Jose Ramón Sendra, ${ }^{2}$ \\ and Laura M. Lechuga ${ }^{1}$ \\ ${ }^{1}$ Nanobiosensors and Bioanalytical Applications Group, Research Center on Nanoscience and Nanotechnology \\ CIN2(CSIC) and CIBER-BBN, 08193 Bellaterra, Barcelona, Spain \\ ${ }^{2}$ IUMA, Institute for Applied Microelectronics, University of Las Palmas GC, Las Palmas GC, Spain
}

(Received 11 October 2012; accepted 11 January 2013; published online 29 January 2013)

\begin{abstract}
We have developed a hybrid platform that combines two well-known biosensing technologies based on quite different transducer principles: surface plasmon resonance and nanomechanical sensing. The new system allows the simultaneous and real-time detection of two independent parameters, refractive index change $(\Delta n)$, and surface stress change $(\Delta \sigma)$ when a biomolecular interaction takes place. Both parameters have a direct relation with the mass coverage of the sensor surface. The core of the platform is a common fluid cell, where the solution arrives to both sensor areas at the same time and under the same conditions (temperature, velocity, diffusion, etc.).The main objective of this integration is to achieve a better understanding of the physical behaviour of the transducers during sensing, increasing the information obtained in real time in one single experiment. The potential of the hybrid platform is demonstrated by the detection of DNA hybridization. () 2013 American Institute of Physics. [http://dx.doi.org/10.1063/1.4789430]
\end{abstract}

\section{INTRODUCTION}

Biosensor technology has become a powerful diagnostic analytical tool. Label-free biosensors are especially interesting because molecules are not altered and can be detected in their natural forms saving time and reagents, and opening the way to implement full integrated and portable systems. Most of the existing biosensing techniques rely on only one single transducer mechanism. According to the transducer principle, biosensors can be roughly classified as: optical, nanomechanical, electrochemical, and piezoelectrical. In general, all of them are valuable techniques capable of covering the continuous demanding requirements at the analytical field. Indeed, biosensors have been successfully employed in a wide range of applications, from genomic and proteomic, to pathogen or single cell detection, with high sensitivity and specificity. However, most biosensors have limitations inherent to their principle of working, where the system sensitivity is limited by the transducer principle and/or the detection method. So far, an option to increase the fields of application and the amount of gather information is the multiparametric detection, either with the detection of a single property of different biological molecules with a single biosensor, the detection of different properties (parameters) of a specific biomolecule with a single platform, or the integration of both of them.

In this work, we show the development of a new platform that implements two of these well-known biosensing technologies: surface plasmon resonance (SPR) and nanomechanical sensors (NM). Both techniques, independently, are still under continuous development for improving their performances by using new physical insights or by new chem-

\footnotetext{
a) Author to whom correspondence should be addressed. Electronic mail: mar.alvarez@cin2.es. Tel.: +34 935814923. Fax: +34 935868020.
}

ical modifications and by broadening their application variety. The working principle and applications of these two individual biosensors have been extensively described in several excellent review papers. ${ }^{1-4}$ Moreover, its individual applications, the SPR has been widely combined with other analytical techniques, such as surface enhanced Raman scattering (SERS), ${ }^{5,6}$ or even atomic force microscopy. ${ }^{7-9}$ As well, the serial and parallel use of SPR with other techniques is a common procedure to complement the bioassays, i.e., SPR-mass spectrometry, ${ }^{10}$ and SPR-microcantilever. ${ }^{11}$ Our method, unlike to Igarashi et al.'s ${ }^{11}$ method, is not a parallel use of both sensors but a implementation of both techniques sharing a common microfluidic chamber where biointeractions will take place over the two sensor surfaces simultaneously.

Our system will record different physical properties of the same biosensing interaction by using both transducer principles. The SPR is a well established technique that provides information about the amount of biomolecules in solution while the microcantilever-based technique provides information about the amount of biomolecules and the forces acting during the process (static mode). This force-related detection mechanism (not reachable with other biosensing techniques) makes NM biosensor a quite unique platform that can provide direct information about conformational changes in biomolecules.

With this configuration, we can measure simultaneously, and in real-time, two independent parameters, the refractive index change, $\Delta n$, and the surface stress change, $\Delta \sigma$, that could contribute with complementary information to a better control and understanding of the surface covering and the biochemical reactions under study. The platform shows several advantages with respect of using both techniques independently, such as sharing a common fluid cell, where the solution arrives to both sensor areas at the same time and under 
the same conditions (temperature, velocity, diffusion, etc.), and a sample volume reduction. At the same time, it is possible to perform a real direct comparison of both techniques, obtaining more information than measuring with both systems independently due to the parameters relation, $\Delta n$ (coverage) versus $\Delta \sigma$ (forces). We demonstrate the potential of this new platform by applying the system to the detection of DNA hybridization.

\section{WORKING PRINCIPLES}

A scheme of the two transducer principles of the proposed biosensing platform is shown in Fig. 1. The SPR sensing mechanism is based on the refractive index changes produced in the adjacent medium of a metal layer, gold in this case. These changes produce a variation on the plasmon propagation vector $\left(k_{\mathrm{sp}}\right)$ generated by a light focused at the interface of the metal and the dielectric layer. The changes in the refractive index could be detected by measuring one of the characteristics of the optical wave interacting with the surface plasmon, angle, wavelength, intensity, phase o polarization. We choose the SPR based on the intensity shifts of the reflected laser beam (maintaining a specific incident angle). During a biorecognition process, the change of the refractive index induced by the analyte molecules binding to the biorecognition layer depends on the volume refractive index increment $(\mathrm{d} n / \mathrm{d} c)_{\mathrm{vol}}$. If the binding occurs within a thin layer over the sensor surface of thickness $h$ the change of the refractive index is related with the surface concentration in

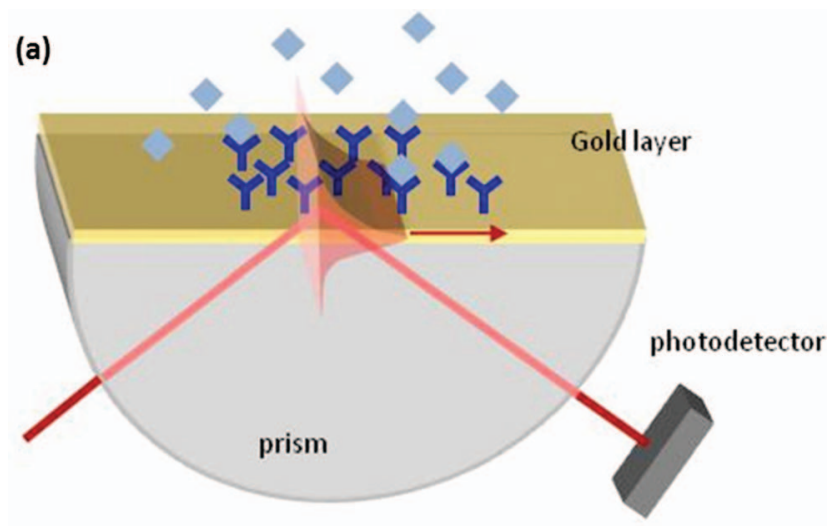

(b)

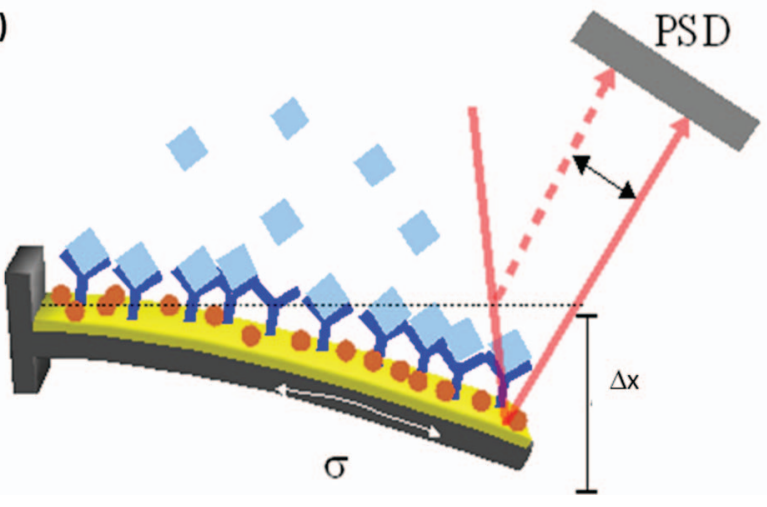

FIG. 1. Schematic representation of the working principles of: (a) SPR and (b) microcantilever based biosensors. mass/area, $\Gamma$, following the equation: ${ }^{12}$

$$
\Delta n_{b}=\left(\frac{d n}{d c}\right)_{\mathrm{vol}} \frac{\Delta \Gamma}{h} .
$$

However, the degree of surface coverage is not easy to determine by using SPR technology, where only a relation between the refractive index change and the reflectivity is given, without studying the relation between the reflectivity and the mass added onto the surface. ${ }^{13}$ Fortunately, the information about the adsorbed mass is not essential to get meaningful results with SPR, being possible to determine the minimum detectable concentration of a specific molecule in solution and achieve kinetic studies without this information.

The working principle of nanomechanical transducers, and specifically of microcantilevers, involves the translation of the biochemical reaction occurring on top of the cantilever surface into a mechanical motion. The induced cantilever response could be a change on the cantilever bending or/and a shift on its resonance frequency. The detection of these responses is usually referred as static and dynamic modes of operation, respectively. Working in the static mode, the sensing mechanism is based on the cantilever bending due to surface stress differences, $\Delta \sigma$, between both cantilever surfaces. Working on the dynamic mode, the sensing mechanism is the shift of the microcantilever frequency, $\Delta f$, due to the total added mass, $\Delta m$, and the microcantilever stiffness change produced by the strain-dependent surface stress (elasticity). This last contribution can cancel or make negligible the resonance frequency change due to the added mass: ${ }^{14}$

$$
\Delta f=\frac{1}{2} f\left(\frac{\Delta k}{k}-\frac{\Delta m^{*}}{m^{*}}\right),
$$

where $f$ is the cantilever fundamental resonance frequency, $m^{*}$ is the effective mass of the cantilever, and $\Delta k$ is the spring constant change. Note that $m^{*}=n m_{\text {beam }}$, where $m_{\text {beam }}$ is the mass of the cantilever and $n=0.24$ for rectangular cantilevers.

Comparing the fundamental resonance frequency due to the surface stress obtained with a string model, and the fundamental resonance frequency of a harmonic oscillator, the change in the cantilever spring constant due to changes in the surface stress is given by ${ }^{15}$

$$
\Delta k=\frac{\pi^{2} n}{4}\left(\Delta \sigma_{1}+\Delta \sigma_{2}\right),
$$

where $\Delta \sigma_{1}$ and $\Delta \sigma_{2}$ are the change in the surface stress on each cantilever surface.

For the development of this platform we chose the static mode working principle because: (i) the cantilever sensitivity only depends on the cantilever spring constant, (ii) the type of information that can be obtained (related to molecules forces), and (iii) the simplicity of the required set-up. Moreover, cantilevers with a low spring constant were used to increase the cantilever bending. ${ }^{4}$ For monitoring the nanomechanical motion induced on the cantilever we used the optical detection method. In the optical lever scheme, the cantilever free end movement is detected by measuring the reflected laser beam displacement into a four quadrant photodetector. A gold layer deposited over one cantilever side increases the laser 
reflection, and reassembles the SPR surface, making possible to apply the same surface chemistry.

The easiest and most extended model to study the surface stress produced on cantilevers is based on Stoney's model, where the total surface stress change between the top and the bottom side $(\Delta \sigma)$ is related with the cantilever free end displacement, $\Delta z$, the Young's modulus, $E$, the Poisson coefficient, $v$, and the cantilever length, $L$, and thickness, $t$, by

$$
\Delta \sigma=\frac{E t^{2}}{3 L^{2}(1-v)} \Delta z
$$

The nanomechanical biosensor based on the surface stress detection has a very complex physical behaviour, and there are currently many open questions about the microcantilever response. The bending of the microcantilever has a nonlinear response with the coverage due to the interactions forces between adsorbed neighboring molecules. Moreover, the microcantilever is a flexible substrate, which means that during the hybridization process the cantilever curvature change can increase or decrease the distance between the neighboring molecules, enhancing or reducing the chains accessibility and the acting forces, modifying the final signal response. The cantilever bending direction and magnitude may depend on the $\mathrm{pH}$ and ionic strength of the solution, giving opposed responses for the same bioreaction performed under lightly different conditions. ${ }^{16,17}$

\section{HYBRID PLATFORM DESCRIPTION}

\section{A. Transducers}

Commercially available silicon nitride rectangular cantilevers from Olympus coated with chromium and gold were used in all the experiments (length, $200 \mu \mathrm{m}$; width, $40 \mu \mathrm{m}$; thickness, $0.8 \mu \mathrm{m}$; nominal spring constant, $0.10 \mathrm{~N} / \mathrm{m}$; density, $\rho=3100 \mathrm{~kg} \mathrm{~m}^{3}$; and Young's modulus $E=1.5 \times 10^{11}$ $\mathrm{N} / \mathrm{m}^{2}$ ). The SPR sensor chip was a microscope glass cover slide $\left(10 \times 10 \times 0.15 \mathrm{~mm}^{3}\right)$ coated with $5 \mathrm{~nm}$ of Ti and $45 \mathrm{~nm}$ of gold. Prior to the experiments, the cantilever and the SPR chip were initially cleaned in organic solvents (trichloroethylene, acetone, and ethanol) heated at $50{ }^{\circ} \mathrm{C}$, rinsed with DI water, and them immersed in fresh made piranha solution for few seconds $\left(96 \% \mathrm{H}_{2} \mathrm{SO}_{4}-30 \% \mathrm{H}_{2} \mathrm{O}_{2}, 3: 1\right)$, rinsed with DI water again and dry under nitrogen flux.

\section{B. Fluid cell}

The core of the system is a PMMA microfluidic cell that contains two independent chambers: one common chamber shared by the cantilever chip and the gold sensor surface of the SPR, and an independent chamber that only covers the SPR gold surface. Fluids are delivered into the chambers by connecting polymeric tubes of $0.6 \mathrm{~mm}$ of diameter to the inlet and outlet channels of each chamber. The independent SPR channel is implemented as an additional reference for checking any cross-effect of the cantilever over the SPR behaviour, as could be laser diffractions onto the SPR gold layer, changes on the SPR signal offset depending on the cantilever bending, or effects in the flow velocity or diffusion inside the cham-

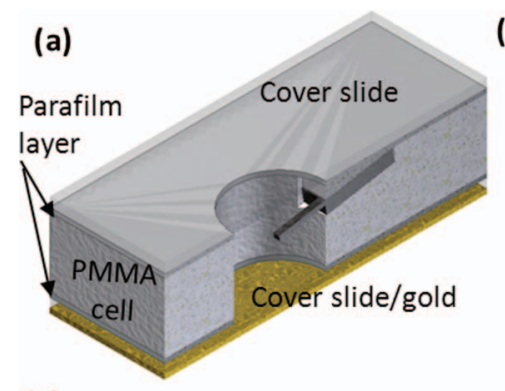

(b)
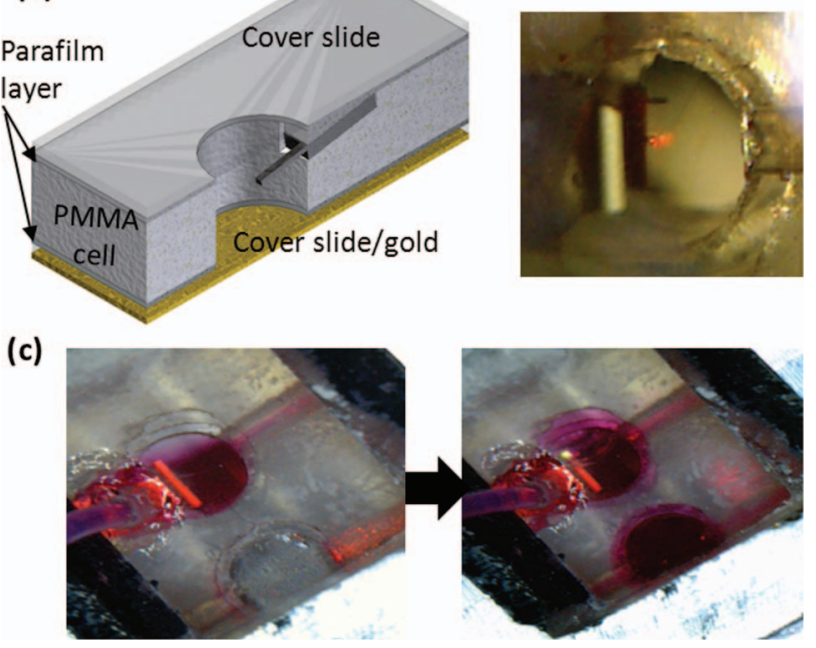

FIG. 2. Fluid cell. (a) Schematic representation of the shared fluid chamber (not at scale). (b) Photograph of the shared chamber (gold surface behind and cantilever in front). (c) Photograph of the injection of a coloured solution into both sensing chambers of the fluid cell.

ber due to the related position between the microcantilever chip and the SPR gold surface (the proximity between both sensors could modify the flow velocity and direction avoiding that molecules could reach the SPR surface). The 3D microfluidic cell consists of five different layers (see Fig. 2(a)): a first $0.15 \mathrm{~mm}$ glass slide covered with gold that will be the SPR sensor surface; a thin layer of PDMS or parafilm $(0.1 \mathrm{~mm})$ with the chambers form for sealing on top to the gold surface; a PMMA cell $(1.0 \mathrm{~mm})$ with two different chambers (one for locating the NM chip and covering the SPR surface, and another one only for covering other region of the SPR surface) and the corresponding inlets and outlets defined by drilling; another thin layer of PDMS or parafilm for sealing and a final glass slide cover $(0.1 \mathrm{~mm})$ to close the chambers. The volume of each chamber is about $1.7 \mathrm{~mm}^{3}$.

With this configuration, the SPR gold surface is situated behind the NM sensor in the shared chamber, as shown in Fig. 2(b). A coloured dilution was used to check the proper interchange of solutions inside the chambers (Fig. 2(c)), and to compare the behaviour of both chambers. We confirm that there are not death regions inside the chambers or diffusion problems and that all the solution inside both chambers is properly interchanged.

To deliver the fluids and the sample solutions, we used a two-channel peristaltic pump (Instech) which allow to maintain a constant flow rate during the experiments on both chambers, two six-port manual injection valves with $250 \mu$ l loop each one, and two injection micro-pumps (Biochem valve) to fill the loops of the valves with the sample solutions for its following injection into the fluid cell, without disturbing the fluid base flow. All the components from the fluidic delivery system are controlled by custom software with a graphical user interface. This configuration enables the introduction of different solutions into each chamber of the fluid cell, allowing the parallel analysis of both sensors surfaces with the same 
biorecognition assay (using one sensor surface as SPR reference) or with a different one.

\section{Optical read-out system}

The instrumentation has two independent optical detection subsystems, one based on the Krestchmam configuration for the SPR measurements and another one based on the optical deflection method for the read-out of the cantilever bending, as shown in Fig. 3(a). Two laser diodes (Edmund optics, $635 \mathrm{~nm}$ ) and two photodetectors, one of two quadrants (S5870, Hamamatsu) and one of four quadrants (S4349, Hamamatsu), for the SPR and cantilever read-out, respectively, were employed. The detection subsystems and all mechanical parts, translation and rotatory stages, were mounted on a $15 \times 15 \mathrm{~cm}^{2}$ aluminium breadboard (Thorlabs) as shown in Fig. 3(b). The fluidic cell, the prism, and the SPR photodetector were mounted over a rotatory stage to find the SPR resonance angle and to fix the angle at which the intensity changes will be detected (Fig. 3(b)). The SPR laser and the rotatory stage were, at the same time, mounted onto a second rotatory stage, which allows modifying the angle of the cantilever reflected laser beam for centering it into the pho-

(a)
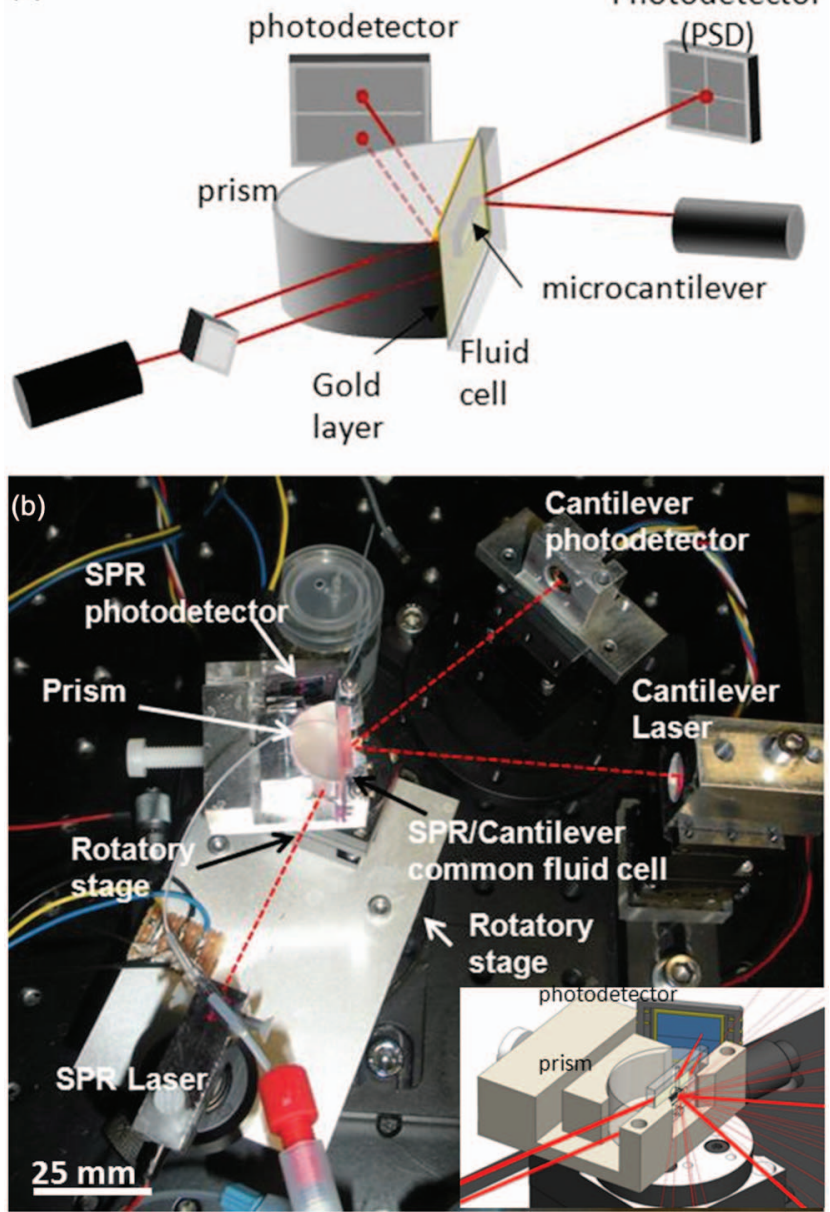

FIG. 3. (a) Configuration scheme of the NM-SPR hybrid sensor. (b) Photograph of the set-up (top-view) and schematic close view of the fluid chamber. todetector area, compensating any cantilever initial offset or shifts in the SPR working angle.

\section{Control system}

We designed a full specific electronic system for the development and implementation of the NM-SPR biosensor. Both the optical read-out systems and the fluid delivery system are managed by a control system. The control system is composed by a custom hardware and software design. The hardware can be divided into several functional blocks (Fig. 4(a)): laser control, injection and peristaltic pumps control, and conditioning and data acquisition. All these blocks are managed by a micro-controller interconnected with a computer by means of a universal serial bus (USB). Custom software manages the setup from the computer. The fluidics diagram block is divided into two parts: power supply and start/stop control of the injections pumps just to introduce the sample in a fast way into the charge loop $(5 \mathrm{~s}$ for a length

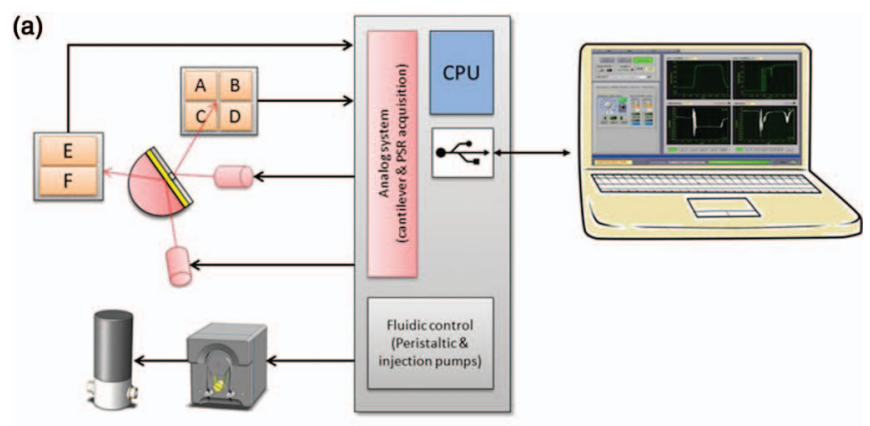

(b)

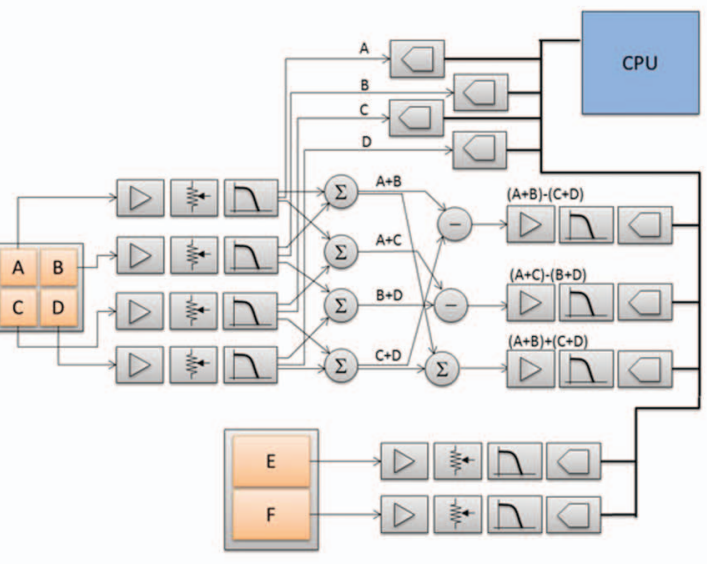

(c)

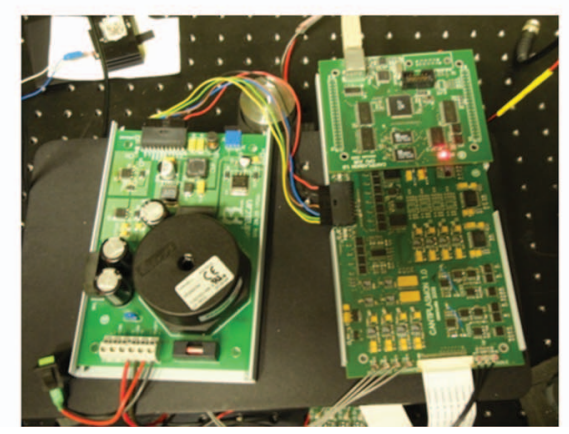

FIG. 4. (a) Diagram block of the hardware. (b) Scheme of the NM-SPR ana$\log$ acquisition system. (c) Fabricated electronic card for the simultaneous acquisition of SPR and NM signals. 
loop of $250 \mu \mathrm{l}$ ), and power supply and speed control of the peristaltic pump to create a constant flow across the fluidic cell.

The control system of the NM-SPR biosensor includes two distinct analog acquisition systems, one for the quadrant sensor photodiode which monitors the cantilever movement and twisting and another dedicated to measuring the reflectance from the SPR sensor (Figs. 4(b) and 4(c)). The complete acquisition system (quadrant and photodiode sensor) uses four identical acquisition schemes, which includes transimpedance amplifiers, low noise and high gain offset compensation circuits to correct the problems caused by the dark current of detectors and electronic offset introduced by the analog model itself. Both, the gain and the offset compensation circuit, are programmable by software, so both the user and the biosensor calibration firmware can adjust these parameters, if requested, for each experiment. Every signal is filtered by anti-aliasing structures before the analog-digital conversion.

To quantify the displacement of the laser beam reflected by the cantilever, we must measure the light that reaches each quadrant photodiode and use those values. That is, the expression $(A+B)-(C+D)$ shows beam vertical displacement and the expression $(\mathrm{A}+\mathrm{C})-(\mathrm{B}+\mathrm{D})$ indicates horizontal displacements.

The numerical calculation of the previous expressions has been discarded after a detailed analysis of quantization errors introduced by the analog-digital converters. The analog implementation of these operations through circuital solutions based on operational and instrumentation amplifiers has been developed to avoid the quantization errors. The digitalization is made at the end of the mathematical operation, extending the dynamic range of the entire system.

The $(\mathrm{A}+\mathrm{B})+(\mathrm{C}+\mathrm{D})$ term is used for monitoring the total optical power received by the quadrant sensor as a reference signal. Any parameter measured will be normalized so to compensate the variations in the laser output power. Similarly, this signal can be used to verify that the beam is within the active area and that has not left the region of interest. This is a mandatory step in the system calibration. All these expressions are digitized and quantified with resolutions of 16 bits. Each channel gain is programmable independently, allowing the circuit to suit different optical configurations that can be done in the experiment, including the change of power and laser type. Four independent inputs are included, corresponding to the separate photodiodes signals, in order to quantify the amount of light received by each photodiode and then to estimate the position of the laser spot in the center of the quadrant sensor. These values are plotted in the control software for guiding the beam centered during the biosensor calibration process.

Moreover, the acquisition electronics (Fig. 4(c)) includes a dual-channel SPR biosensor based on a dual photodiode (E and F) with analog circuitry and signal conditioning programmable gain. These circuits are responsible for measuring variations in the irradiance of the signals produced by the SPR photodiodes. The acquisition is performed also at 16 bits after an appropriate filtering step. The control software is divided into two distinct sections, one that is built into the CPU of

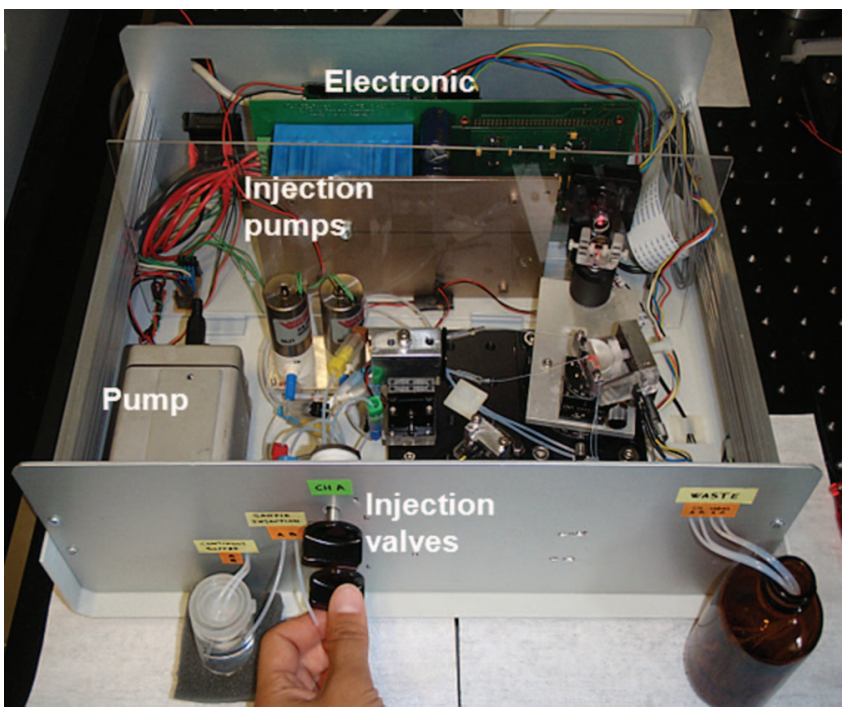

FIG. 5. Portable prototype of the hybrid platform.

the electronic system (firmware) and another section located in the PC. The firmware manages the programming and monitoring of all parameters for calibration and operation of the biosensor. All of these commands are received from the USB interface via graphical user application.

\section{E. Packaging of the hybrid platform}

The different sub-systems that constitute the hybrid platform (the fluid cell, the read-out systems, and the fluid delivery system) were packaged into a case to get a portable biosensing platform (see Fig. 5). Housing the set-up reduces the interference of the external light on the photodetectors as well.

\section{RESULTS}

\section{A. Characterization}

The SPR bulk refractive index sensitivity was characterized by injecting dilutions of ethanol, which produce a change in the bulk refractive index but with no significant adsorption on the sensor surface. Dilutions of ethanol at 25\%, $10 \%$, $5 \%, 1 \%, 0.5 \%$, and $0.1 \%$, with refractive indexes previously evaluated with a commercial Abbe refractometer (1.3465 for ethanol 25\%), were injected. The stabilization solution was DI water, with a refractive index of 1.3325 . The SPR response is shown in Fig. 6(a) (top)-higher ethanol concentrations are not represented. The experimental bulk detection limit of the SPR sensor is $\Delta n=5.5 \times 10^{-5}$, corresponding to an ethanol dilution of $0.1 \%$. Concentrations of ethanol lower than $0.1 \%$ are difficult to sense due to the noise of the system of about $0.15 \%$ ( 3 times standard deviation of the base line). Fig. 6(b) shows the good linearity of the response achieved with the SPR sensor, which is one of the critical parameters in the biosensor sensitivity. Each data are a mean value of four different experiments.

The injection of ethanol dilutions also helps for checking the effect of refractive index changes on the cantilever 
(a)

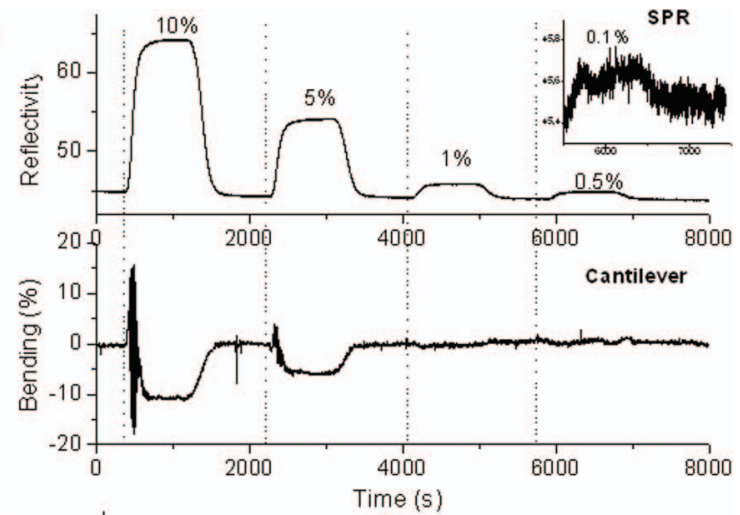

(b)

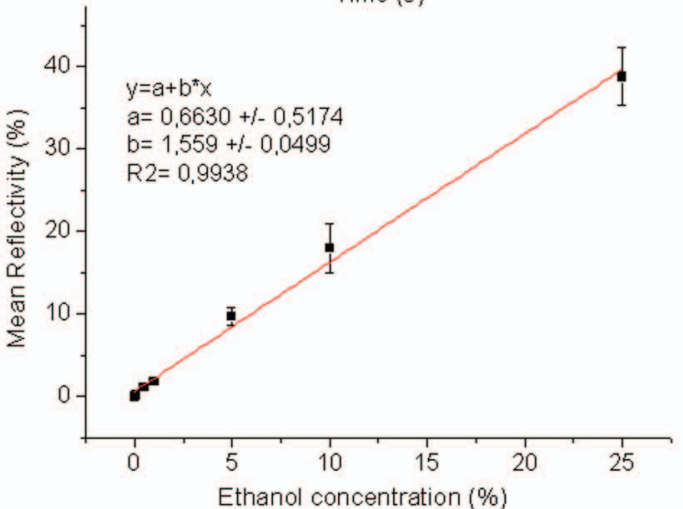

(c)

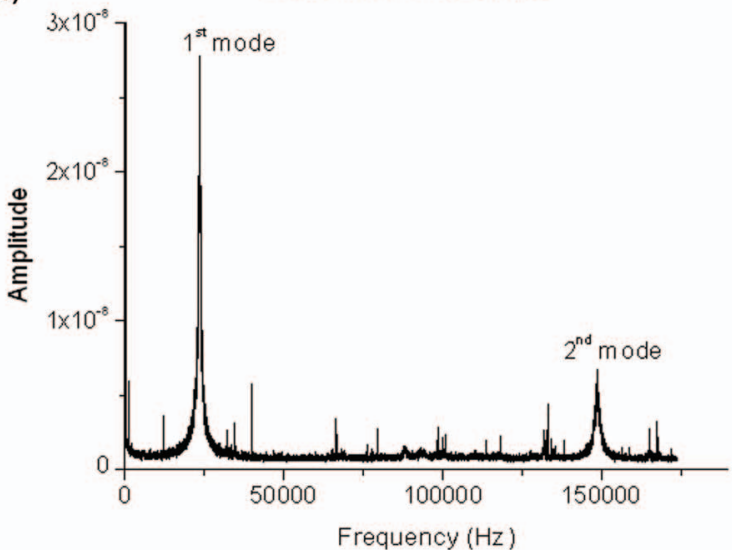

FIG. 6. (a) Simultaneous acquisition of SPR and NM signals for the injection of different dilutions of ethanol in water. The inset shows the minimum dilution of ethanol measurable with our home-made SPR $\left(\Delta n \sim 5 \times 10^{-5}\right)$. (b) Linear fitting of the mean intensity evaluated by SPR vs ethanol concentrations. (c) Thermal noise power spectrum of the cantilever.

response by the real-time monitoring of both SPR and cantilever signals. Due to the shift in the laser pathway when the refractive index of the surrounding medium changes, the cantilever response corresponding to the biointeraction can be modified or hided. ${ }^{18}$ The simultaneously monitored changes in the SPR and the cantilever signals due to the injection of ethanol dilutions are shown in Fig. 6(a) (bottom), where it can be observed that changes in the refractive index of $4 \times 10^{-4}$ (and lower) slightly affect the cantilever reflected beam. Most of the biological solutions of interest are used at concentrations which refractive index would not affect the cantilever response; therefore we can conclude that the proposed cantilever configuration is optimal for assessing biomolecular interactions.
For characterizing the NM sensor, we used the geometrical method and measured the thermal noise power spectrum. In the NM sensor, the displacement of the laser spot on the photodetector, $\Delta d$, can be related to the cantilever bending, $\Delta x(\mathrm{~nm})$, using geometrical methods as in Eq. (5), where $s$ is the distance from cantilever to the photodetector, and $L$ is the cantilever length:

$$
\Delta x=\frac{\Delta d L}{4 s},
$$

$\Delta d(\mathrm{~nm})$ depends on the photodetector sensitivity and can be written as $\Delta d=10^{6}\left(I_{1}-I_{2}\right) l_{\mathrm{psd}} / 2\left(I_{1}+I_{2}\right)$, where $l_{\mathrm{psd}}$ is the active photodetector length in $\mathrm{mm}, I_{1}-I_{2}$ is the differential signal and $I_{1}+I_{2}$ is the sum signal. For our particular set-up ( $L=200 \mu \mathrm{m}$ and $s=100 \mathrm{~mm}$ ) and substituting $\Delta d$ into (5), a deflection factor $\left(L 10^{6} / 4 s\right)$ of 500 is obtained. ${ }^{19}$

Beside the geometrical calibration, a measurement of the cantilever resonance frequency was performed by acquiring the power spectrum due to the cantilever thermal noise without additional external amplification of the cantilever vibration. The detection of the cantilever movement due to the thermal noise implies that the nanomechanical system configuration has nanometric resolution, since cantilever thermal vibration is in the $\mathrm{nm}$ order. The cantilever movement was measured via the voltage difference between the right and left half of the photodetector, and the fast Fourier transform (FFT) of this signal gives the cantilever power spectrum shown in Fig. 6(c), observing the first and the second cantilever resonance mode. The cantilever resonance frequency is measured before each experiment to check the cantilever integrity and the system resolution. However, we do not detect the cantilever resonance frequency during the bio-recognition process. The reason is that we do not use any external amplification of the cantilever vibration motion and when working in liquid, the frequency resolution is not enough to detect the small changes of the frequency.

The final sensitivity for a specific biomolecule detection will be given by the system resolution plus the effect of external factors such as fluid diffusion, surface coverage, or the physiological medium employed (buffer type, $\mathrm{pH}$, salt concentration, etc.), as discussed in Sec. III.

\section{B. DNA hybridization assay}

To evaluate the performance of the hybrid platform of a biorecognition process, we conducted DNA hybridization assays. The nanomechanical detection of the DNA hybridization has attracted a high amount of attention due to the complex cantilever response found, depending on governing forces playing different roles. ${ }^{20-28}$ Optimized conditions for enhancing the detection when working with DNA molecules are difficult to set as a general rule because these conditions have a high dependency on the specific sensor surface. Recent publication demonstrates the hybridization efficiency at different $\mathrm{pH}$, reaching a peak for high $\mathrm{pH} .{ }^{17}$ Most of the reported works use external complementary techniques, such as fluorescent, radiolabelling or SPR, to quantify the surface coverage and to optimize the working conditions of NM sensors. The novel system that we proposed is a suitable 
platform for applications where the cantilever response is difficult to interpret and the use of complementary techniques, such as SPR, could play a crucial role for understanding what is really happening over the sensor surface.

In our experiments, previously to the hybridization detection, we carry out the formation of a single stranded DNA (ssDNA) probe monolayer covalently bonded onto the gold surfaces. For that purpose, the DNA single strand sequence used for immobilization was a 24-mer that bears a thiol linker (C6-SH) at the $5^{\prime}$ end (sh-ssDNA). This allows using the selfassembled monolayer chemistry on both sensor surfaces (SPR and NM). The immobilization of DNA probes was carried out at constant flow rate; using $1 \mu \mathrm{M}$ of the sample prepared on a volume of $250 \mu \mathrm{l}$ in $50 \mathrm{mM}$ phosphate buffered (PB) solution with $750 \mathrm{mM} \mathrm{NaCl}$, at $\mathrm{pH}$ 7. Hybridization experiments were done at room temperature using a target volume of $250 \mu \mathrm{l}$ in the same buffer, at a concentration of $500 \mathrm{nM}$. The complementary sequence was a 32-mer single stranded DNA with a free tail at the $3^{\prime}$ end after hybridizing with the DNA probes. For surface regeneration, formamide $35 \%$ was employed.

The simultaneous monitoring of both sensing signals, SPR and NM, during the in-flow immobilization of the DNA probes is shown in Fig. 7(a) (top) and (bottom), respectively. In the beginning, the chambers were filled with the same buffer that will be used for the immobilization process, at a constant flow rate, until equilibrium in the drift of the cantilever bending was reached. Then, by using the injections valves automatically controlled by software, the $250 \mu \mathrm{l}$ of the ssDNA probes solution was injected without stopping or modifying the flow rate. The equilibrium buffer will reach and wash the chambers after $20 \mathrm{~min}$, removing the unbound DNA chains from the sensors surfaces. In this case, the SPR signal was acquired from both independent chambers/areas, demonstrating that there is no effect of the cantilever over the SPR response. The light difference in the kinetic of both SPR signals is attributed to a difference in the cleaning stage of both sensing regions. The formation of the self-assembled monolayer of DNA produces a downward deflection of the microcantilever (the gold side is expanding respect to the silicon side). The chosen $\mathrm{pH} 7$ produces a decrease in the steric interaction between neighboring ssDNA strands and a higher electrostatic repulsive forces, bending the microcantilever away from the gold surface to provide more space for the target DNA.

The subsequent hybridization with the complementary chains produces a net differential signal in both sensors, as can be observed in Fig. 7(b). The microcantilever response is again a downward bending (compressive stress), due to the increase of the electrostatic forces between the double stranded DNA chains. The injection of a control solution $(250 \mu \mathrm{l}$ of non-complementary 32-mer ssDNA at a concentration of $500 \mathrm{nM}$ ) does not produce any NM or SPR net signal changes. The change in the SPR signal due to the injection of the non-complementary DNA chains is only due to bulk refractive index changes as can be deduced because the base line is recovered again once the equilibrium buffer washes the chambers. This result demonstrates the specificity of both sensors surfaces.
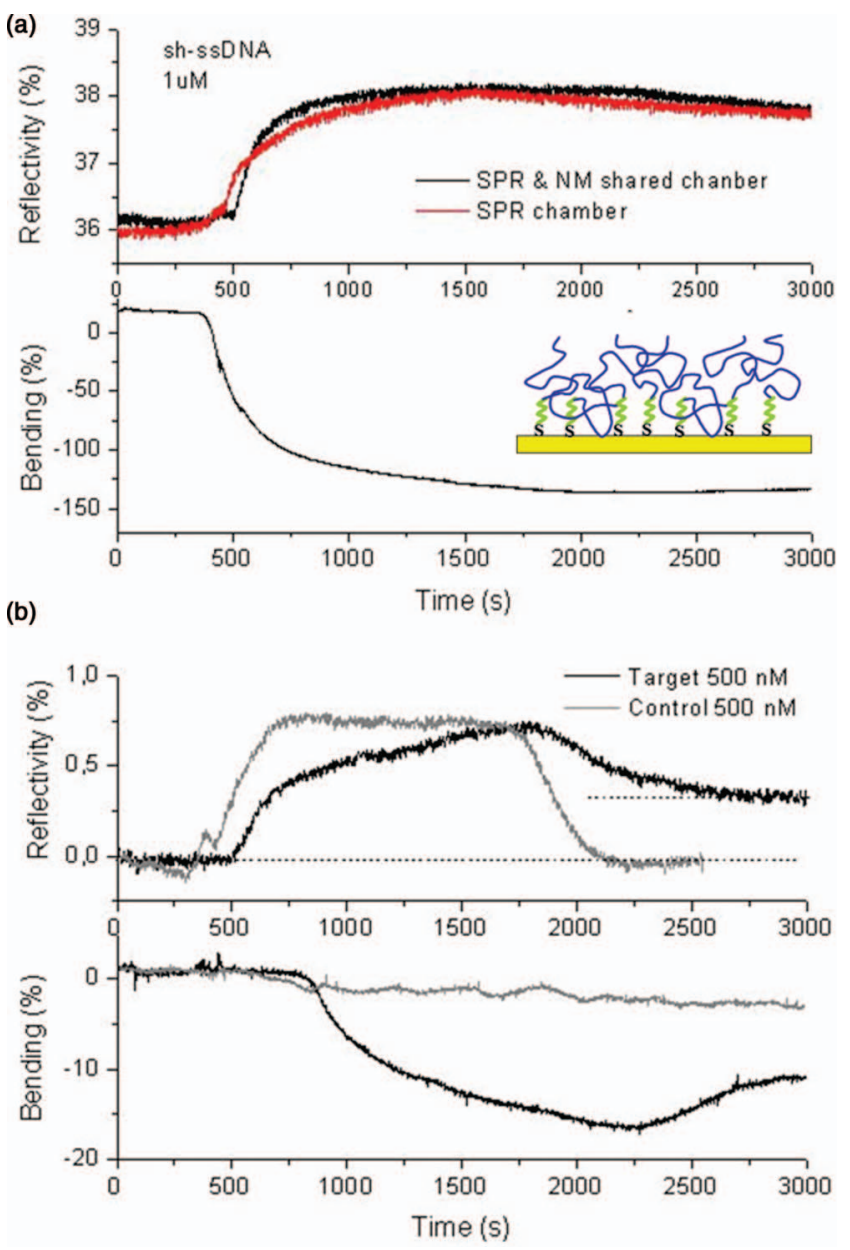

FIG. 7. Simultaneous acquisition of SPR and NM signals for the in-flow immobilization of sh-ssDNA (a) and the subsequent ssDNA hybridization and control experiments (b).

The bending signal evaluated nanomechanically during the hybridization process corresponds to a surface stress change of $13.44 \mathrm{mN} / \mathrm{m}$ (calculated by using Eq. (4)). This value is in accordance with previously reported data for hybridization experiments. The change on the surface stress produces a change on the cantilever spring constant, which at the same, affects the cantilever resonance frequency. Although this frequency variation does not have any effect on the biorecognition detection when working in the static mode, it may be interesting to discuss the associated frequency change produced during our experiments. Considering a surface covering of $\sim 10^{12}$ molecules $/ \mathrm{cm}^{2}$, the maximum added mass to the cantilever during the hybridization experiments is $\sim 0.6 \mathrm{pg} .{ }^{29}$ The effect on the resonance frequency due to this added mass and the cantilever stiffness change can be calculated by using Eqs. (2) and (3). The added mass ratio, $\Delta m / m$, would be $\sim 1.2 \times 10^{-4}$ (for a cantilever mass of about $20 \mathrm{ng}$ ), while the spring constant ratio, $\Delta k / k$, would be of $\sim 0.077$ (for a spring constant change of $0.0077 \mathrm{~N} / \mathrm{m}$ and a cantilever spring constant of $0.1 \mathrm{~N} / \mathrm{m}$ ). Following Eq. (2), the total change in the resonance frequency would be $\sim 800 \mathrm{~Hz}$, where the spring constant change would be the governing parameter in opposition to the mass change. 


\section{CONCLUSIONS}

A new hybrid platform was evaluated for the simultaneous monitoring of two independent parameters during a biorecognition process, the surface stress and the refractive index change, by using two different biosensing principles. We have demonstrated the potential of the hybrid integrated platform by the detection of DNA hybridization. The cantilever-based biosensor is still a promising technique under development. A complete understanding of the surface stress origin is essential to optimize the bending method, to reduce the limit of detection, and for obtaining more information about the biological interaction. The simultaneous and direct comparison with a well established technique, such as SPR, can contribute to this understanding. Some improvements could be done in the future to reduce the SPR noise and to increase the bulk experimental sensitivity by including a thermal control, reducing the laser divergence, or increasing the working wavelength. Future developments of the hybrid platform will be focused on the system scalability and the addition of another sensing parameter: the cantilever resonance frequency change. This will provide an interesting correlation between three parameters: $\Delta n$, mass, and surface stress. We pursue to develop a multichannel platform with one or several microcantilevers per channel and one SPR per channel, with independent flow injections for each channel, which will lead to a multiplexed and multiparametric biosensor.

\section{ACKNOWLEDGMENTS}

Authors acknowledge the financial support from Juan de la Cierva Program (MICINN-JDC) and Fundación M. Botín.

${ }^{1}$ J. Homola, Chem. Rev. 108, 462 (2008).

${ }^{2}$ X. Fan, I. M. White, S. I. Shopova, H. Zhu, J. D. Suter, and Y. Sun, Anal. Chim. Acta 620, 8 (2008).

${ }^{3}$ M. N. Velasco-Garcia, Semin. Cell Dev. Biol. 20, 27 (2009).

${ }^{4}$ M. Alvarez and L. M. Lechuga, Analyst 135, 827 (2010).
${ }^{5}$ S. Nie and S. R. Emory, Science 275, 1102 (1997).

${ }^{6}$ S. A. Meyer, E. C. Le Ru, and P. G. Etchegoin, Anal. Chem. 83, 2337 (2011).

${ }^{7}$ A. Baba, W. Knoll, and R. Advincula, Rev. Sci. Instrum. 77, 064101 (2006).

${ }^{8}$ J. Davies, C. J. Roberts, A. C. Dawkes, J. Sefton, J. C. Edwards, T. O. Glasbey, A. G. Haymes, M. C. Davies, and D. E. Jackson, Langmuir 10, 2654 (1994).

${ }^{9}$ K. M. Shakesheff, X. Chen, M. C. Davies, A. Domb, C. J. Roberts, S. J. B. Tendler, and P. M. Williams, Langmuir 11, 3921 (1995).

${ }^{10}$ C. Williams and T. A. Addona, Trends Biotechnol. 18, 45 (2000).

${ }^{11}$ S. Igarashi, A. N. Itakura, M. Toda, M. Kitajima, L. Chu, A. N. Chifen, R. Förch, and R. Berger, Sens. Actuators B 117, 43 (2006).

${ }^{12}$ J. Homola, Surface Plasmon Resonance Based Sensors (Springer, 2006).

${ }^{13}$ P. Englebienne, A. Van Hoonacker, and M. Verhas, Spectroscopy 17, 255 (2003).

${ }^{14}$ B. N. Johnson and R. Mutharasan, Biosens. Bioelectron. 32, 1 (2012).

${ }^{15}$ G. Y. Chen, T. Thundat, E. A. Wachter, and R. J. Warmack, J. Appl. Phys. 77, 3618 (1995).

${ }^{16}$ M. L. Sushko, J. H. Harding, A. L. Shluger, R. A. McKendry, and M. Watari, Adv. Mater. 20, 3848 (2008).

${ }^{17}$ J. Zhang, H. P. Lang, G. Yoshikawa, and C. Gerber, Langmuir 28, 6494 (2012).

${ }^{18}$ Y. Huang, H. Liu, K. Li, Y. Chen, Q. Zhang, and X. Wu, Sens. Actuators A 148, 329 (2008).

${ }^{19}$ R. Mishra, W. Grange, and M. Hegner, J. Sensors 2012, 1 (2012).

${ }^{20}$ N.-H. Zhang, J.-Z. Chen, J.-J. Li, and Z.-Q. Tan, Biomaterials 31, 6659 (2010).

${ }^{21}$ R. Mukhopadhyay, M. Lorentzen, J. Kjems, and F. Besenbacher, Langmuir 21, 8400 (2005)

${ }^{22}$ J. C. Stachowiak, M. Yue, K. Castelino, A. Chakraborty, and A. Majumdar, Langmuir 22, 263 (2006).

${ }^{23}$ M. F. Hagan and A. K. Chakraborty, J. Chem. Phys. 120, 4958 (2004).

${ }^{24}$ J. Fritz, M. K. Baller, H. P. Lang, H. Rothuizen, P. Vettiger, E. Meyer, H.-J. Güntherodt, C. Gerber, and J. K. Gimzewski, Science 288, 316 (2000).

${ }^{25}$ G. Wu, H. Ji, K. Hansen, T. Thundat, R. Datar, R. Cote, M. F. Hagan, A. K. Chakraborty, and A. Majumdar, Proc. Natl. Acad. Sci. U.S.A. 98, 1560 (2001)

${ }^{26}$ R. McKendry, J. Zhang, Y. Arntz, T. Strunz, M. Hegner, H. P. Lang, M. K. Baller, U. Certa, E. Meyer, H.-J. Güntherodt, and C. Gerber, Proc. Natl. Acad. Sci. U.S.A. 99, 9783 (2002).

${ }^{27}$ M. Alvarez, L. G. Carrascosa, M. Moreno, A. Calle, A. Zaballos, L. M. Lechuga, C. Martinez-A, and J. Tamayo, Langmuir 20, 9663 (2004).

${ }^{28}$ H. P. Lang, M. Hegner, E. Meyer, and C. Gerber, Nanotechnology 13, R29 (2002).

${ }^{29}$ S. Zheng, J. H. Choi, S. M. Lee, K. S. Hwang, S. K. Kim, and T. S. Kim, Lab Chip 11, 63 (2011). 